\title{
High-sensitivity troponin I for risk stratification in normotensive pulmonary embolism
}

\author{
Matthias Ebner ${ }^{1,2}$, Niklas Guddat ${ }^{3}$, Karsten Keller $\mathbb{1}^{4,5}$, \\ Marie Christine Merten ${ }^{3}$, Markus H. Lerchbaumer ${ }^{6}$, Gerd Hasenfuß ${ }^{3,7}$, \\ Stavros V. Konstantinides $\mathbb{1}^{4,8}$ and Mareike Lankeit (10) $2,3,4,9$
}

Affiliations: ${ }^{1}$ Dept of Cardiology and Angiology, Campus Charité Mitte (CCM), Charité - University Medicine Berlin, Berlin, Germany. ${ }^{2}$ German Center for Cardiovascular Research (DZHK), partner site Berlin, Germany. ${ }^{3}$ Clinic of Cardiology and Pneumology, University Medical Center Göttingen, Göttingen, Germany. ${ }^{4}$ Center for Thrombosis and Hemostasis (CTH), University Medical Center Mainz, Johannes Gutenberg-University Mainz, Mainz, Germany. ${ }^{5}$ Center for Cardiology, Cardiology I, University Medical Center Mainz, Johannes GutenbergUniversity Mainz, Mainz, Germany. ${ }^{6}$ Dept of Radiology, CCM, Charité - University Medicine Berlin, Berlin, Germany. ${ }^{7}$ German Center for Cardiovascular Research (DZHK), partner site Goettingen, Germany. ${ }^{8}$ Dept of Cardiology, Democritus University of Thrace, Alexandroupolis, Greece. ${ }^{9}$ Dept of Internal Medicine and Cardiology, Campus Virchow Klinikum (CVK), Charité - University Medicine Berlin, Berlin, Germany.

Correspondence: Mareike Lankeit, Dept of Internal Medicine and Cardiology, CVK, Charité - University Medicine Berlin, Augustenburger Platz 1, 13353 Berlin, Germany. E-mail: mareike.lankeit@acharite.de

ABSTRACT While numerous studies have confirmed the prognostic role of high-sensitivity troponin $\mathrm{T}$ (hsTnT) in pulmonary embolism (PE), high-sensitivity troponin I (hsTnI) is inappropriately studied. This study aimed to investigate the prognostic relevance of hsTnI in normotensive PE, establish the optimal cut-off value for risk stratification and to compare the prognostic performances of hsTnI and hsTnT.

Based on data from 459 consecutive PE patients enrolled in a single-centre registry, receiver operating characteristic analysis was used to identify an optimal hsTnI cut-off value for prediction of in-hospital adverse outcomes (PE-related death, cardiopulmonary resuscitation or vasopressor treatment) and allcause mortality.

Patients who suffered an in-hospital adverse outcome (4.8\%) had higher hsTnI concentrations compared with those with a favourable clinical course (57 (interquartile range (IQR) 22-197) versus 15 (IQR 10-86) $\mathrm{pg} \cdot \mathrm{mL}^{-1}, \mathrm{p}=0.03$ ). A hsTnI cut-off value of $16 \mathrm{ng} \cdot \mathrm{mL}^{-1}$ provided optimal prognostic performance and predicted in-hospital adverse outcomes (OR 6.5, 95\% CI 1.9-22.4) and all-cause mortality (OR 3.7, 95\% CI 1.0-13.3). Between female and male patients, no relevant differences in hsTnI concentrations (17 (IQR 10-97) versus 17 (IQR 10-92) pg. $\mathrm{mL}^{-1}, \mathrm{p}=0.79$ ) or optimised cut-off values were observed. Risk stratification according to the 2019 European Society of Cardiology algorithm revealed no differences if calculated based on either hsTnI or hsTnT $(\mathrm{p}=0.68)$.

Our findings confirm the prognostic role of hsTnI in normotensive PE. HsTnI concentrations $>16 \mathrm{pg} \cdot \mathrm{mL}^{-1}$ predicted in-hospital adverse outcome and all-cause mortality; sex-specific cut-off values do not seem necessary. Importantly, our results suggest that hsTnI and hsTnT can be used interchangeably for risk stratification.

@ERSpublications

The study confirms the prognostic relevance of high-sensitivity troponin $I$ in normotensive pulmonary embolism. A cut-off value of $16 \mathrm{pg} \cdot \mathrm{mL}^{-1}$ can be used for risk stratification in male and female patients; sex-specific adjustments do not appear necessary. https://bit.ly/3lCECip

Cite this article as: Ebner M, Guddat N, Keller K, et al. High-sensitivity troponin I for risk stratification in normotensive pulmonary embolism. ERJ Open Res 2020; 6: 00625-2020 [https:// doi.org/10.1183/23120541.00625-2020].

This article has supplementary material available from openres.ersjournals.com.

Received: 28 Aug 2020 | Accepted: 6 Oct 2020

Copyright $\odot$ ERS 2020. This article is open access and distributed under the terms of the Creative Commons Attribution Non-Commercial Licence 4.0. 


\section{Introduction}

Pulmonary embolism (PE) is a life-threatening disease with high morbidity and mortality $[1,2]$. Current guideline recommendations emphasise the importance of early risk stratification in the heterogeneous group of normotensive patients with acute PE to guide therapeutic decision making [3].

Consensus exists that right ventricular (RV) dysfunction is the critical determinant of outcome in PE [3]. In the absence of chronic pulmonary hypertension, the RV has a narrow range to handle an acute increase in afterload caused by the occlusion of pulmonary vasculature during acute PE [4]. If the adaptive potential of the RV is exceeded, RV dilation and elevated wall tension occurs $[5,6]$. Consequently, coronary perfusion of the RV myocardium is impaired, leading to ischaemia that further aggravates RV dysfunction and ultimately cardiac failure [6]. Thus, detection of myocardial injury indicated by cardiac troponin elevation is a key element for identification of normotensive patients at higher risk for short-term adverse outcomes [3, 7-9].

High-sensitivity troponin assays have largely replaced conventional assays due to their increased ability to detect myocardial injury [10]. While a large number of studies confirmed the prognostic relevance of high-sensitivity troponin $\mathrm{T}$ (hsTnT) in acute PE and established cut-off values defining elevated hsTnT concentrations $[8,11]$, only a single study investigated high-sensitivity troponin I (hsTnI) for risk stratification in a small cohort of 65 unselected PE patients [12]. Therefore, the prognostic relevance of hsTnI elevation in patients with acute PE is inappropriately studied and disease-specific cut-off values remain undefined.

In the present study we aimed to investigate the prognostic relevance of hsTnI, establish the optimal hsTnI cut-off value and compare the prognostic performances of hsTnI and hsTnT for risk stratification of normotensive patients with acute PE.

\section{Methods}

Study design and definition of outcomes

The Pulmonary Embolism Registry of Göttingen (PERGO) prospectively includes consecutive patients with objectively confirmed PE $\geqslant 18$ years of age admitted to the University Medical Center Göttingen, Germany. The study protocol has been described in detail previously $[13,14]$. Briefly, patient recruitment is performed by daily screening of new admissions to the emergency department and of reports of conducted computed tomography pulmonary angiographies (CTPAs). After obtaining informed consent for participation in PERGO, complete data on comorbidities, previous medication, symptoms, results from diagnostic tests (including laboratory parameters, ECG, transthoracic echocardiography (TTE) and CTPA), treatment and clinical course is obtained using a standardised case report form. In addition, adjudication of parameters defining RV dysfunction is performed by an independent investigator by reviewing the electronically stored TTE and CTPA images. The present analysis included patients enrolled in PERGO between September 2008 and April 2018. We excluded patients with: 1) missing hsTnI measurements at presentation; 2) cardiac arrest or haemodynamic instability at presentation; and 3) another acute cardiorespiratory illness such as acute myocardial infarction, left heart decompensation or respiratory decompensation responsible for clinical presentation and symptoms. All patients were followed for the in-hospital stay.

Diagnostic and therapeutic management was in accordance with the European Society of Cardiology (ESC) $2008(09 / 2008-08 / 2014)$ and $2014(09 / 2014-04 / 2018)$ guidelines [15, 16] and local standard operating procedures. All related decisions were left to the discretion of the treating physicians and were not influenced by the study protocol. Treating physicians were not informed about study results, thus any influence of the study on patient management or monitoring of treatment effects can be excluded. The study was conducted in accordance with the amended Declaration of Helsinki and approved by the local independent Ethic Committee of the University Medical Center Göttingen, Germany. All patients gave informed written consent for participation in the study.

Tachycardia was defined as heart rate of $\geqslant 100$ beats per minute $(\mathrm{bpm})$ and hypoxaemia as peripheral oxygen saturation $<90 \%$. Renal insufficiency was defined as glomerular filtration rate (GFR) $<60 \mathrm{~mL} \cdot \mathrm{min}^{-1} \cdot 1.73 \mathrm{~m}^{-2}$ body surface area. Altered mental status was defined as disorientation, somnolence, sopor or coma. Active cancer was defined as known malignant disease, treatment with antitumour therapy within the last 6 months, metastatic state or haematologic cancer not in complete remission [17]. RV dysfunction on CTPA was defined as right-to-left ventricular (RV/LV) diameter ratio $\geqslant 1.0$ [3]. Patients were stratified to risk classes post hoc according to the algorithm proposed by the ESC 2019 guidelines and the modified FAST score $[3,9]$. The modified FAST score includes elevated troponin (1.5 points), tachycardia ( 2 points) and syncope ( 1.5 points); a patient is classified as high risk if $\geqslant 3$ points are scored. For calculation of all algorithms and scores, missing values were considered to be normal [18]. 
An in-hospital adverse outcome was defined as PE-related death or haemodynamic collapse (cardiopulmonary resuscitation or administration of catecholamines). The second study outcome was in-hospital all-cause mortality. Death was determined to be PE-related if either confirmed by autopsy or following a clinically severe episode of acute PE in absence of an alternative diagnosis. All events and causes of death were independently adjudicated by two of the authors (M.E. and N.G.) and disagreement was resolved by a third author (M.L.).

\section{Biomarker measurements}

Venous blood samples were collected on admission or at the moment of PE diagnosis, processed using standard operating procedures and immediately stored at $-80^{\circ} \mathrm{C}$. Plasma concentrations of hsTnI (ARCHITECT stat hsTnI assay, Abbott Laboratories, Chicago, IL, USA), hsTnT (Roche Diagnostics, Mannheim, Germany) and N-terminal pro-brain natriuretic peptide (NT-proBNP; Roche Diagnostics, Mannheim, Germany) were measured in batches after a single thaw by the amedes MVZ wagnerstibbe laboratory in Göttingen, Germany. For hsTnI, the following predefined cut-off values were investigated: 1) $>10 \mathrm{pg} \cdot \mathrm{mL}^{-1}$ (lower detection limit of the assay); 2) $>27 \mathrm{pg} \cdot \mathrm{mL}^{-1}$ (99th percentile in healthy individuals) [19]; 3) $>16 \mathrm{pg} \cdot \mathrm{mL}^{-1}$ in women and $>34 \mathrm{pg} \cdot \mathrm{mL}^{-1}$ in men (sex-specific 99 th percentile in healthy individuals) [20]; and 4) $>100 \mathrm{pg} \cdot \mathrm{mL}^{-1}$ (previously evaluated for risk stratification in acute PE) [12]. For other biomarkers, cut-off values indicating elevated concentrations were prospectively defined as hsTnT $\geqslant 14 \mathrm{pg} \cdot \mathrm{mL}^{-1}$ [18] and NT-proBNP $\geqslant 600 \mathrm{pg} \cdot \mathrm{mL}^{-1}$ [21]. Serial biomarker measurements were not performed.

\section{Statistical analysis}

Categorical variables are presented as total numbers and percentages and continuous variables are presented as medians with interquartile ranges (IQR). Associations between binary and categorical variables were analysed using Fisher's exact test, Chi-squared test or the Mantel-Haenszel test of trend, as appropriate. Spearman's rank correlation coefficient was used to test for statistical dependence of hsTnI from continuous variables. For comparison of continuous variables, the Mann-Whitney U-test was employed.

To evaluate the overall prognostic performance of hsTnI with regard to study outcomes, receiver operating characteristic (ROC) curve analyses were performed to determine the area under the curve (AUC) with corresponding 95\% confidence intervals (95\% CI) in the whole study cohort and in patients stratified according to sex. The optimal cut-off value for prediction of study outcomes was identified based on Youden index quantification and compared to the established hsTnI and hsTnT cut-off values.

Further, the prognostic value of selected cut-off values, patient characteristics, comorbidities and biomarkers with regard to study outcomes was tested using univariable logistic regression analyses and results are presented as odds ratios (ORs) with the corresponding 95\% CI. To confirm the independent prognostic value of hsTnI, hsTnI levels dichotomised according to the optimal cut-off value were in entered in three multivariable logistic regression models correcting for: 1) presence of coronary artery disease; 2) renal insufficiency; and 3 ) age $\geqslant 75$ years.

To test for differences in the prognostic performance of the ESC 2019 algorithm and the modified FAST score if calculated based on either hsTnT or hsTnI levels, the net reclassification improvement (NRI) with corresponding SE was calculated [22]. Kaplan-Meier analysis was used to compare the probability of 1-year survival in subgroups stratified according to hsTnI levels at presentation; the log-rank test was used for comparison between groups.

A two-sided significance level of $\alpha<0.05$ was defined as appropriate to indicate statistical significance. As this was explorative testing, no adjustments for multiple testing were carried out. The p-values were provided for descriptive reasons only and should be interpreted with caution and in connection with effect estimates. Statistical analysis was performed through Statistics Package for Social Sciences (IBM SPSS Statistics, version 26, IBM Corp. Armonk, NY, USA).

\section{Results}

Between September 2008 and April 2018, 854 patients were enrolled in PERGO. Exclusion criteria applied to: 1) 283 patients with missing hsTnI measurements; 2) 88 patients with cardiogenic shock or haemodynamic instability at presentation; and 3) 24 patients with significant acute cardiorespiratory illness responsible for clinical presentation and symptoms. Hence, 459 patients (53.7\%) were included in the present analysis. A comparison of the study cohort with patients excluded due to missing hsTnI measurements is provided in the supplementary material (table S1). 
At presentation, $58(12.6 \%)$ patients were classified as low risk, $265(57.7 \%)$ as intermediate-low risk and $136(29.6 \%)$ as intermediate-high risk according to the ESC 2019 risk-stratification algorithm. Reperfusion treatment was performed in $10(2.2 \%)$ patients and $7(1.5 \%)$ were enrolled in the PEITHO trial [23]. An in-hospital adverse outcome occurred in $22(4.8 \%)$ patients. Overall, 14 (3.1\%) patients died during the in-hospital stay; of those, $9(64.3 \%)$ deaths were due to PE. Information on baseline characteristics, comorbidities, initial presentation and outcomes in are shown in table 1, left column.

The median biomarker concentrations on admission were 17.0 (IQR 9.9-96.0) $\mathrm{pg} \cdot \mathrm{mL}^{-1}$ for hsTnI, 20.0 (IQR 8.7-56.7) $\mathrm{pg} \cdot \mathrm{mL}^{-1}$ for hsTnT and 499 (IQR 108-2386) $\mathrm{pg} \cdot \mathrm{mL}^{-1}$ for NT-proBNP. More specifically, $41.6 \%$ of patients had hsTnI concentrations $>27 \mathrm{pg} \cdot \mathrm{mL}^{-1}$ and $23.5 \%$ of patients $>100 \mathrm{pg} \cdot \mathrm{mL}^{-1}$, while elevated hsTnT concentrations $\geqslant 14 \mathrm{pg} \cdot \mathrm{mL}^{-1}$ were observed in $61.0 \%$ of patients. A strong correlation between hsTnI and hsTnT concentrations $(\mathrm{r}=0.82, \mathrm{p}<0.001)$ was observed. Furthermore, hsTnI concentrations showed a positive correlation with age $(\mathrm{r}=0.31, \mathrm{p}<0.001)$ and heart rate $(\mathrm{r}=0.20, \mathrm{p}<0.001)$ and an inverse correlation with GFR $(\mathrm{r}=-0.32, \mathrm{p}<0.001)$.

\section{Identification of the optimal hsTnl cut-off value for risk stratification}

Patients who developed an in-hospital adverse outcome had higher hsTnI concentrations compared to patients with a favourable clinical course $\left(57\right.$ (IQR 22-197) $\mathrm{pg} \cdot \mathrm{mL}^{-1}$ versus 15 (IQR 10-86) $\mathrm{pg} \cdot \mathrm{mL}^{-1}$, $\mathrm{p}=0.030$ ). Further, the rate of in-hospital adverse outcomes increased with higher hsTnI concentrations at presentation ( $\mathrm{p}=0.002$ for trend; supplementary figure $\mathrm{S} 1$ ).

TABLE 1 Comorbidities, results from risk stratification and outcomes of study patients stratified according to the optimal hsTnl cut-off value

\begin{tabular}{|c|c|c|c|c|}
\hline & All patients & $\mathrm{hsTnl}>16 \mathrm{pg} \cdot \mathrm{mL}^{-1}$ & $\mathrm{hsTnl} \leqslant 16 \mathrm{pg} \cdot \mathrm{mL}^{-1}$ & p-value \\
\hline Subjects $\mathrm{n}$ & 459 & 234 & 225 & \\
\hline Age $\geqslant 75$ years & $155(33.8 \%)$ & $102(43.6 \%)$ & $53(23.6 \%)$ & $<0.001$ \\
\hline \multicolumn{5}{|l|}{ Comorbidities } \\
\hline Chronic heart failure & $59(12.9 \%)$ & $43(18.4 \%)$ & $16(7.1 \%)$ & $<0.001$ \\
\hline Coronary artery disease & $67(14.6 \%)$ & $46(19.7 \%)$ & $21(9.3 \%)$ & 0.002 \\
\hline Renal insufficiency & $134(29.2 \%)$ & $98(41.9 \%)$ & $36(16.0 \%)$ & $<0.001$ \\
\hline Active cancer & $110(24.0 \%)$ & $45(19.2 \%)$ & $65(28.9 \%)$ & 0.015 \\
\hline \multicolumn{5}{|l|}{ Symptoms at presentation } \\
\hline Dyspnoea & $359(78.6 \%), n=457$ & $190(81.9 \%), n=232$ & $169(75.1 \%)$ & 0.08 \\
\hline Syncope & $53(11.6 \%), n=457$ & $39(16.8 \%), n=232$ & $14(6.2 \%)$ & $<0.001$ \\
\hline Altered mental status & $19(4.1 \%), n=458$ & $11(4.7 \%), n=233$ & $8(3.6 \%)$ & 0.53 \\
\hline $\mathrm{hsTnT} \geqslant 14 \mathrm{pg} \cdot \mathrm{mL}^{-1}$ & $259(60.2 \%), n=430$ & $196(92.9 \%), n=211$ & $63(28.8 \%), n=219$ & $<0.001$ \\
\hline $\mathrm{NT}-$ proBNP $\geqslant 600 \mathrm{pg} \cdot \mathrm{mL}^{-1}$ & $212(47.5 \%), n=446$ & $168(75.3 \%), n=223$ & $44(19.7 \%), n=223$ & $<0.001$ \\
\hline RV/LV diameter ratio $\geqslant 1.0$ on CTPA & $270(65.4 \%), n=413$ & $163(79.5 \%), n=205$ & $107(51.4 \%), n=208$ & $<0.001$ \\
\hline \multicolumn{5}{|l|}{ Risk stratification } \\
\hline Modified FAST score $\geqslant 3$ points ${ }^{\#}$ & $115(25.1 \%)$ & $113(48.3 \%)$ & $2(0.9 \%)$ & $<0.001$ \\
\hline ESC 2019 algorithm ${ }^{\#}$ & & & & $<0.001$ \\
\hline Low risk & $58(12.6 \%)$ & $0(0.0 \%)$ & $58(25.8 \%)$ & \\
\hline Intermediate-low risk & $265(57.7 \%)$ & $98(41.9 \%)$ & $167(74.2 \%)$ & \\
\hline Intermediate-high risk & $136(29.6 \%)$ & $136(58.1 \%)$ & $0(0.0 \%)$ & \\
\hline \multicolumn{5}{|l|}{ Outcome } \\
\hline In-hospital adverse outcome & $22(4.8 \%)$ & $19(8.1 \%)$ & $3(1.3 \%)$ & $<0.001$ \\
\hline Catecholamine administration & 15 (3.3\%) & $13(5.6 \%)$ & $2(0.9 \%)$ & 0.005 \\
\hline Cardiopulmonary resuscitation & $5(1.1 \%)$ & $5(2.1 \%)$ & $0(0.0 \%)$ & 0.027 \\
\hline
\end{tabular}

Data are presented as $\mathrm{n}(\%)$, unless otherwise stated. HsTnl: high-sensitivity troponin I; hsTnT: high-sensitivity troponin $\mathrm{T}$; NT-proBNP: $\mathrm{N}$-terminal pro-brain natriuretic peptide; RV/LV: right/left ventricle; CTPA: computed tomography pulmonary angiography; ESC: European Society of Cardiology; PE: pulmonary embolism. " : Calculation of scores based on hsTnl measurements. 
FIGURE 1 Receiver operating characteristics curve of hsTnl concentrations with regard to an adverse in-hospital outcome. Bullet points indicate different investigated high-sensitivity troponin I (hsTnl) cut-off values.

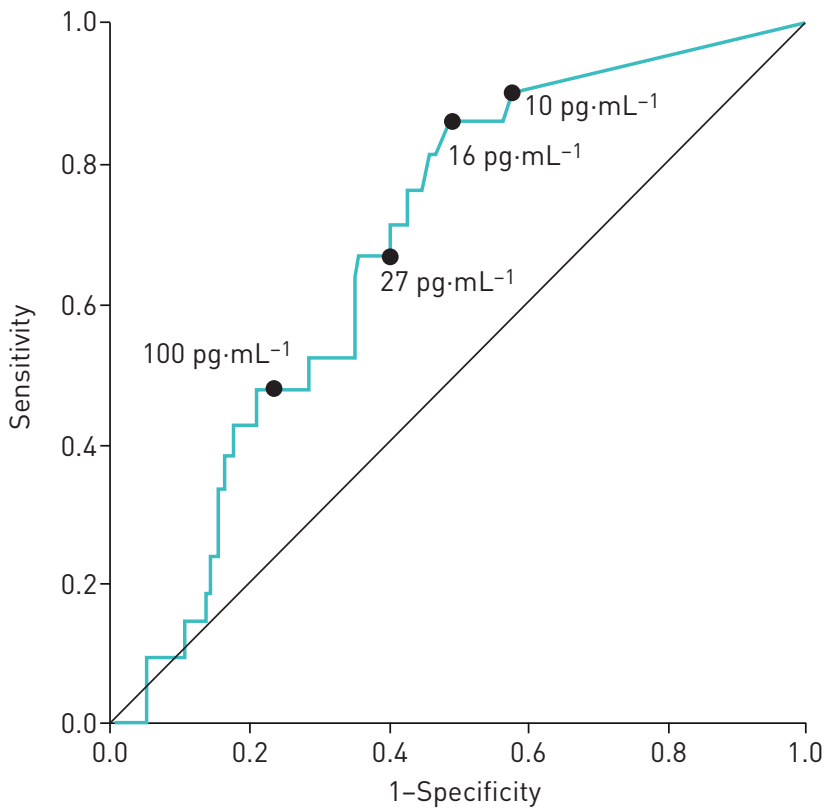

ROC analyses indicated an association between hsTnI and in-hospital adverse outcome (AUC 0.68 (95\% CI 0.59-0.77); figure 1) and in-hospital all-cause mortality (AUC 0.64 (95\% CI 0.51-0.77)). Using Youden index quantification, an optimal cut-off value of $17 \mathrm{pg} \cdot \mathrm{mL}^{-1}$ for the identification of patients with an in-hospital adverse outcomes was identified. As this value is numerically almost identical to the 99th percentile of hsTnI concentrations in healthy women $\left(16 \mathrm{pg} \cdot \mathrm{mL}^{-1}\right)$ and Youden's indices for the two cut-off values differed only minimally ( 0.38 and 0.37 , respectively), we selected a cut-off value of $16 \mathrm{pg} \cdot \mathrm{mL}^{-1}$ for all further analysis.

Patients with hsTnI levels $>16 \mathrm{pg} \cdot \mathrm{mL}^{-1}$ were older, had higher rates of chronic heart failure, coronary artery disease and renal insufficiency as well as higher rates of dyspnoea, syncope, tachycardia, hypoxaemia and signs of RV dysfunction on diagnostic imaging compared to patients with hsTnI levels $\leqslant 16 \mathrm{pg} \cdot \mathrm{mL}^{-1}$ (table 1 , right columns).

\section{Evaluation of sex-specific differences}

No difference in the median hsTnI concentrations between female and male PE patients (17.0 (IQR 9.9-97.2) versus 16.5 (IQR 9.9-92.3) $\mathrm{pg} \cdot \mathrm{mL}^{-1}, \mathrm{p}=0.79$ ) was observed and Youden index quantification indicated similar optimal hsTnI cut-off values for both sexes $\left(17 \mathrm{pg} \cdot \mathrm{mL}^{-1}\right.$ for female and $19 \mathrm{pg} \cdot \mathrm{mL}^{-1}$ for male patients). However, a larger AUC with regard to an in-hospital adverse outcome was calculated for female $(0.74$ (95\% CI 0.63-0.85)) than for male patients (0.62 (95\% CI 0.47-0.76)).

Prognostic performance of the optimal hsTnl cut-off value

Using univariable logistic regression analysis, hsTnI $>16 \mathrm{pg} \cdot \mathrm{mL}^{-1}$ predicted an in-hospital adverse outcome (OR 6.5 (95\% CI 1.9-22.4); table 2, middle columns) as well as all-cause mortality (OR 3.7 (95\% CI 1.0-13.3); table 2, right columns). The independent prognostic value of hsTnI with regard to the primary outcome was confirmed in three separate multivariable models, correcting for the presence of: 1) coronary artery disease (OR 6.5 (95\% CI 1.9-22.6)); 2) renal insufficiency (OR 4.7 (95\% CI 1.3-16.6)); or $3)$ age $\geqslant 75$ years (OR 6.0 (95\% CI 1.7-20.9))

A comparison of test characteristics for the newly identified PE-specific cut-off value of $16 \mathrm{pg} \cdot \mathrm{mL}^{-1}$ and all predefined hsTnI and hsTnT cut-off values is provided in table 3.

Prognostic performance of risk stratification based on hsTnT or hsTnl measurements

A comparison of the prognostic performance of the modified FAST score and the ESC 2019 algorithm calculated based on: 1) hsTnI $>16 \mathrm{pg} \cdot \mathrm{mL}^{-1}$ and 2) $\mathrm{hsTnT} \geqslant 14 \mathrm{pg} \cdot \mathrm{mL}^{-1}$ is provided in table 4 . Calculations of sensitivity, specificity and ORs for outcome prediction did not reveal relevant differences in test performance. Further, no difference in the NRI was observed. 
TABLE 2 Predictors of in-hospital adverse outcome and all-cause mortality

\begin{tabular}{|c|c|c|c|c|c|c|c|}
\hline & \multirow[t]{2}{*}{ All patients } & \multicolumn{3}{|c|}{ In-hospital adverse outcome } & \multicolumn{3}{|c|}{ In-hospital all-cause mortality } \\
\hline & & Yes & No & OR $(95 \% \mathrm{Cl})$ & Yes & No & OR $(95 \% \mathrm{CI})$ \\
\hline Subjects $\mathrm{n}$ & 459 & 22 & 437 & & 14 & 445 & \\
\hline Age $\geqslant 75$ years & $155(33.8 \%)$ & $11(50.0 \%)$ & $144(33.0 \%)$ & $2.03(0.86-4.8)$ & $7(50.0 \%)$ & $148(33.3 \%)$ & $2.01(0.69-5.83)$ \\
\hline Sex (female) & $241(52.5 \%)$ & $11(50.0 \%)$ & $230(52.6 \%)$ & $0.90(0.38-2.12)$ & $9(64.3 \%)$ & $232(52.1 \%)$ & $1.65(0.55-5.01)$ \\
\hline \multicolumn{8}{|l|}{ Comorbidities } \\
\hline Chronic heart failure & $59(12.9 \%)$ & $4(18.2 \%)$ & $55(12.6 \%)$ & $1.54(0.50-4.73)$ & $3(21.4 \%)$ & $56(12.6 \%)$ & $1.89(0.51-7.00)$ \\
\hline Coronary artery disease & $67(14.6 \%)$ & $4(18.2 \%)$ & $63(14.4 \%)$ & $1.32(0.43-4.03)$ & $3(21.4 \%)$ & $64(14.4 \%)$ & $1.62(0.44-5.98)$ \\
\hline Chronic pulmonary disease & $60(13.1 \%), n=458$ & $3(14.3 \%), n=21$ & $57(13.0 \%)$ & $1.11(0.32-3.89)$ & $4(30.8 \%), n=13$ & $56(12.6 \%)$ & $3.09(0.92-10.36)$ \\
\hline Diabetes mellitus & $65(14.2 \%)$ & $4(18.2 \%)$ & $61(14.0 \%)$ & $1.37(0.45-4.18)$ & $3(21.4 \%)$ & $62(13.9 \%)$ & $1.68(0.46-6.21)$ \\
\hline Renal insufficiency & $134(29.2 \%)$ & $14(63.6 \%)$ & $120(27.5 \%)$ & $4.62(1.89-11.30)$ & $8(57.1 \%)$ & $126(28.3 \%)$ & $3.38(1.15-9.92)$ \\
\hline Active cancer & $110(24.0 \%)$ & $5(22.7 \%)$ & $105(24.0 \%)$ & $0.93(0.34-2.58)$ & $7(50.0 \%)$ & $103(23.1 \%)$ & $3.32(1.14-9.69)$ \\
\hline \multicolumn{8}{|l|}{ Symptoms at presentation } \\
\hline Dyspnoea & $359(78.6 \%), n=457$ & $17(81.0 \%), n=21$ & $342(78.4 \%), n=436$ & $1.17(0.38-3.55)$ & $11(84.6 \%), n=13$ & $348(78.4 \%), n=444$ & $1.52(0.33-6.96)$ \\
\hline Syncope & $53(11.6 \%), n=457$ & $6(28.6 \%), n=21$ & $47(10.8 \%), n=436$ & $3.31(1.23-8.95)$ & $3(23.1 \%), n=13$ & $50(11.3 \%), n=444$ & $2.36(0.63-8.88)$ \\
\hline Altered mental status & $19(4.1 \%), n=458$ & $5(23.8 \%), n=21$ & $14(3.2 \%)$ & $9.44(3.03-29.42)$ & $3(23.1 \%), n=13$ & $16(3.6 \%)$ & $8.04(2.02-32.08)$ \\
\hline \multicolumn{8}{|l|}{ Clinical findings at presentation } \\
\hline Tachycardia & $135(30.4 \%), n=444$ & $12(57.1 \%), n=21$ & $123(29.1 \%), n=423$ & $3.25(1.34-7.91)$ & $6(46.2 \%), n=13$ & $129(29.9 \%), n=431$ & $2.01(0.66-6.09)$ \\
\hline Hypoxaemia & 74 (19.8\%), n=374 & $7(33.3 \%), n=21$ & $67(19.0 \%), n=353$ & $2.13(0.83-5.49)$ & $3(23.1 \%), n=13$ & 71 (19.7\%), n=361 & $1.23(0.33-4.57)$ \\
\hline \multicolumn{8}{|l|}{ Laboratory and imaging markers } \\
\hline hsTnl >10 pg.mL $\mathrm{mL}^{-1}$ & $279(60.8 \%)$ & $20(90.9 \%)$ & $259(59.3 \%)$ & 6.87 (1.59-29.77) & $12(85.7 \%)$ & $267(60.0 \%)$ & $4.00(0.89-18.09)$ \\
\hline $\mathrm{hsTnl}>16 \mathrm{pg} \cdot \mathrm{mL}^{-1}$ & $234(51.0 \%)$ & 19 (86.4\%) & $215(49.2 \%)$ & $6.54(1.91-22.42)$ & $11(78.6 \%)$ & $223(50.1 \%)$ & $3.65(1.00-13.26)$ \\
\hline $\mathrm{hsTnl}>27 \mathrm{pg} \cdot \mathrm{mL}^{-1}$ & $191(41.6 \%)$ & $15(68.2 \%)$ & $176(40.3 \%)$ & $3.18(1.27-7.95)$ & $9(64.3 \%)$ & $182(40.9 \%)$ & $2.60(0.86-7.89)$ \\
\hline $\begin{array}{l}\mathrm{hsTnl}>16 \mathrm{pg} \cdot \mathrm{mL}^{-1} \text { (women), } \\
>34 \mathrm{pg} \cdot \mathrm{mL}^{-1} \text { (men) }\end{array}$ & $209(45.5 \%)$ & $17(77.3 \%)$ & $192(43.9 \%)$ & $4.34(1.57-11.97)$ & $11(78.6 \%)$ & $198(44.5 \%)$ & $4.57(1.26-16.62)$ \\
\hline $\mathrm{hsTnl}>100 \mathrm{pg} \cdot \mathrm{mL}^{-1}$ & $108(23.5 \%)$ & 10 (45.5\%) & 98 (22.4\%) & $2.89(1.21-6.87)$ & $6(42.9 \%)$ & 102 (22.9\%) & $2.52(0.86-7.44)$ \\
\hline $\mathrm{hsTnT} \geqslant 14 \mathrm{pg} \cdot \mathrm{mL}^{-1}$ & $271(61.0 \%), n=444$ & $20(95.2 \%), n=21$ & $251(59.3 \%), n=423$ & $13.71(1.82-103.08)$ & $12(85.7 \%)$ & $259(60.2 \%), n=430$ & $3.96(0.88-17.92)$ \\
\hline NT-proBNP $\geqslant 600 \mathrm{pg} \cdot \mathrm{mL}^{-1}$ & $212(47.5 \%), n=446$ & $18(85.7 \%), n=21$ & $194(45.6 \%), n=425$ & $7.14(2.07-24.62)$ & $12(85.7 \%)$ & $200(46.3 \%), n=432$ & $6.96(1.54-31.47)$ \\
\hline RV/LV diameter ratio $\geqslant 1.0$ on CTPA & $270(65.4 \%), n=413$ & $11(64.7 \%), n=17$ & $259(65.4 \%), n=396$ & $0.97(0.35-2.68)$ & $8(80.0 \%), n=10$ & $262(65.0 \%), n=403$ & $2.15(0.45-10.27)$ \\
\hline
\end{tabular}

OR: odds ratio; $\mathrm{Cl}$ : confidence interval; hsTnl: high-sensitivity troponin I; hsTnT: high-sensitivity troponin $\mathrm{T}$; NT-proBNP: N-terminal pro-brain natriuretic peptide; RV/LV: right/left ventricle; CTPA: computed tomography pulmonary angiography. 
TABLE 3 Prognostic performance of different troponin cut-off values

\begin{tabular}{|c|c|c|c|c|c|c|c|c|c|c|c|c|c|c|c|}
\hline Cut-off value & Prevalence & $\begin{array}{l}\text { In-hospital } \\
\text { adverse } \\
\text { outcome }\end{array}$ & $\begin{array}{l}\text { Sensitivity } \\
(95 \% \mathrm{CI})\end{array}$ & $\begin{array}{l}\text { Specificity } \\
(95 \% \text { CI) }\end{array}$ & $\begin{array}{c}\text { PPV } \\
(95 \% \mathrm{Cl})\end{array}$ & $\begin{array}{c}N P V \\
(95 \% \mathrm{Cl})\end{array}$ & LR+ & $\begin{array}{c}\text { OR } \\
(95 \% \mathrm{Cl})\end{array}$ & $\begin{array}{l}\text { In-hospital } \\
\text { all-cause } \\
\text { mortality }\end{array}$ & $\begin{array}{l}\text { Sensitivity } \\
(95 \% \mathrm{CI})\end{array}$ & $\begin{array}{l}\text { Specificity } \\
(95 \% \text { CI) }\end{array}$ & $\begin{array}{l}\text { PPV } \\
(95 \% \text { CI) }\end{array}$ & $\begin{array}{c}\text { NPV } \\
(95 \% \mathrm{Cl})\end{array}$ & LR+ & $\begin{array}{c}\text { OR } \\
(95 \% \mathrm{Cl})\end{array}$ \\
\hline $\begin{array}{l}\text { hs } T \mathbf{n l}>10 \mathrm{pg} \cdot \mathrm{mL}^{-1} \\
\text { (lower limit of detection) }\end{array}$ & $60.8 \%$ & $7.2 \%$ & $\begin{array}{c}90.9 \% \\
(72.2-97.5)\end{array}$ & $\begin{array}{c}40.7 \% \\
(36.2-45.4)\end{array}$ & $\begin{array}{c}7.2 \% \\
(4.7-10.8)\end{array}$ & $\begin{array}{c}98.9 \% \\
(96.0-99.7)\end{array}$ & 1.5 & $\begin{array}{c}6.87 \\
(1.59-29.77)\end{array}$ & $4.3 \%$ & $\begin{array}{c}85.7 \% \\
(60.1-96)\end{array}$ & $\begin{array}{c}40.0 \% \\
(35.6-44.6)\end{array}$ & $\begin{array}{c}4.3 \% \\
(2.5-7.4)\end{array}$ & $\begin{array}{c}98.9 \% \\
(96.0-99.7)\end{array}$ & 1.4 & $\begin{array}{c}4.00 \\
(0.89-18.09)\end{array}$ \\
\hline $\begin{array}{l}\text { hsTnl >16 pg } \cdot \mathrm{mL}^{-1} \\
\text { (optimal cut-off value) }\end{array}$ & $51.0 \%$ & $8.1 \%$ & $\begin{array}{c}86.4 \% \\
(64.0-96.4)\end{array}$ & $\begin{array}{c}50.8 \% \\
(46.0-55.6)\end{array}$ & $\begin{array}{c}8.1 \% \\
(5.3-12.3)\end{array}$ & $\begin{array}{c}98.7 \% \\
(96.2-99.5)\end{array}$ & 1.8 & $\begin{array}{c}6.54 \\
(1.91-22.42)\end{array}$ & $4.7 \%$ & $\begin{array}{c}78.6 \\
(48.9-94.3)\end{array}$ & $\begin{array}{c}49.9 \% \\
(45.2-54.6)\end{array}$ & $\begin{array}{c}4.7 \% \\
(2.6-8.2)\end{array}$ & $\begin{array}{c}98.7 \% \\
(96.2-99.5)\end{array}$ & 1.6 & $\begin{array}{c}3.65 \\
(1.01-13.26)\end{array}$ \\
\hline $\begin{array}{l}\text { hsTnl >27 pg } \cdot \mathrm{mL}^{-1} \\
\text { (99th percentile URL) }\end{array}$ & $41.6 \%$ & $7.9 \%$ & $\begin{array}{c}68.2 \% \\
(47.3-83.6)\end{array}$ & $\begin{array}{c}59.7 \% \\
(55.1-64.2)\end{array}$ & $\begin{array}{c}7.9 \% \\
(4.8-12.6)\end{array}$ & $\begin{array}{c}97.4 \% \\
(94.7-98.7)\end{array}$ & 1.7 & $\begin{array}{c}3.18 \\
(1.27-7.95)\end{array}$ & $4.7 \%$ & $\begin{array}{c}64.3 \% \\
(38.8-83.7)\end{array}$ & $\begin{array}{c}59.1 \% \\
(54.5-63.6)\end{array}$ & $\begin{array}{c}4.7 \% \\
(2.5-8.7)\end{array}$ & $\begin{array}{c}98.1 \% \\
(95.7-99.2)\end{array}$ & 1.6 & $\begin{array}{c}2.60 \\
(0.86-7.89)\end{array}$ \\
\hline $\begin{array}{l}\text { hsTnl >16 pg } \cdot \mathrm{mL}^{-1} \\
\text { (women), >34 pg } \cdot \mathrm{mL}^{-1} \\
\text { (men) } \\
\text { (sex-specific 99th } \\
\text { percentile URL) }\end{array}$ & $45.5 \%$ & $8.1 \%$ & $\begin{array}{c}77.3 \% \\
(54.2-91.3)\end{array}$ & $\begin{array}{c}56.1 \% \\
(51.3-60.8)\end{array}$ & $\begin{array}{c}8.1 \% \\
(5.1-12.6)\end{array}$ & $\begin{array}{c}98.0 \% \\
(95.4-99.1)\end{array}$ & 1.8 & $\begin{array}{c}4.34 \\
(1.57-11.97)\end{array}$ & $5.3 \%$ & $\begin{array}{c}78.6 \\
(48.8-94.3)\end{array}$ & $\begin{array}{c}55.5 \% \\
(50.7-60.2)\end{array}$ & $\begin{array}{c}5.3 \% \\
(3.0-9.2)\end{array}$ & $\begin{array}{c}98.8 \% \\
(96.5-99.6)\end{array}$ & 1.8 & $\begin{array}{c}4.57 \\
(1.26-16.62)\end{array}$ \\
\hline $\begin{array}{l}\text { hsThl }>100 \mathrm{pg} \cdot \mathrm{mL}^{-1} \\
\text { (cut-off value proposed } \\
\text { by [12]) }\end{array}$ & $23.5 \%$ & $9.3 \%$ & $\begin{array}{c}45.5 \% \\
(26.9-65.3)\end{array}$ & $\begin{array}{c}77.6 \% \\
(73.4-81.2)\end{array}$ & $\begin{array}{c}9.3 \% \\
(5.1-16.2)\end{array}$ & $\begin{array}{c}96.6 \% \\
(94.1-98)\end{array}$ & 2.0 & $\begin{array}{c}2.89 \\
(1.21-6.87)\end{array}$ & $5.6 \%$ & $\begin{array}{c}42.9 \% \\
(21.4-67.4)\end{array}$ & $\begin{array}{c}77.1 \% \\
(73-80.7)\end{array}$ & $\begin{array}{c}5.6 \% \\
(2.6-11.6)\end{array}$ & $\begin{array}{c}97.7 \% \\
(95.6-98.8)\end{array}$ & 1.9 & $\begin{array}{c}2.52 \\
(0.86-7.44)\end{array}$ \\
\hline hsTnT $\geqslant 14 \mathrm{pg} \cdot \mathrm{mL}^{-1}$ & $61.0 \%$ & $7.4 \%$ & $\begin{array}{c}95.2 \% \\
(74.1-.99 .8)\end{array}$ & $\begin{array}{c}40.7 \% \\
(36.0-45.6)\end{array}$ & $\begin{array}{c}7.4 \% \\
(4.8-11.1)\end{array}$ & $\begin{array}{c}99.4 \% \\
(96.8-99.9)\end{array}$ & 1.6 & $\begin{array}{c}13.71 \\
(1.82-103.08)\end{array}$ & $4.4 \%$ & $\begin{array}{c}85.7 \% \\
(56.2-97.5)\end{array}$ & $\begin{array}{c}39.8 \% \\
(35.1-44.6)\end{array}$ & $\begin{array}{c}4.4 \% \\
(2.6-7.6)\end{array}$ & $\begin{array}{c}98.8 \% \\
(95.9-99.7)\end{array}$ & 1.4 & $\begin{array}{c}3.96 \\
(0.88-17.92)\end{array}$ \\
\hline
\end{tabular}

Cl: confidence interval; PPV: positive predictive value; NPV: negative predictive value; LR+: positive likelihood ratio; OR: odds ratio; hsTnl: high-sensitivity troponin I; URL: upper reference limit; hsTnT: high-sensitivity troponin T.

TABLE 4 Comparison of risk stratification algorithms calculated based on $\mathrm{hsTnl}$ and hsTnT

\begin{tabular}{|c|c|c|c|c|c|c|c|c|c|c|}
\hline Risk stratification algorithm & $\begin{array}{l}\text { Troponin assay and } \\
\text { cut-off value }\end{array}$ & Prevalence & $\begin{array}{l}\text { In-hospital } \\
\text { adverse outcome }\end{array}$ & $\begin{array}{l}\text { Sensitivity } \\
(95 \% \text { CI) }\end{array}$ & $\begin{array}{l}\text { Specificity } \\
(95 \% \text { CI) }\end{array}$ & $\begin{array}{c}\text { PPV } \\
(95 \% \text { CI) }\end{array}$ & $\begin{array}{c}\text { NPV } \\
(95 \% \text { Cl) }\end{array}$ & LR+ & OR $(95 \% \mathrm{Cl})$ & NRI \\
\hline \multirow[t]{2}{*}{$\begin{array}{l}\text { Modified FAST score } \\
\geqslant 3 \text { points }\end{array}$} & $\mathrm{hs} T \mathrm{TT} \geqslant 14 \mathrm{pg} \cdot \mathrm{mL}^{-1}$ & $21.8 \%$ & $12.0 \%$ & $\begin{array}{c}54.5 \% \\
(32.7-74.9)\end{array}$ & $\begin{array}{c}79.9 \% \\
(75.7-83.5)\end{array}$ & $\begin{array}{c}12.0 \% \\
(7.0-19.8)\end{array}$ & $\begin{array}{c}97.2 \% \\
(94.9-98.5)\end{array}$ & 2.7 & $\begin{array}{c}4.76 \\
(1.99-11.37)\end{array}$ & $\begin{array}{c}0.06 \text { [SE } \\
0.09 \text { ), } p=0.51\end{array}$ \\
\hline & hsTnl >16 pg $\cdot \mathrm{mL}^{-1}$ & $25.1 \%$ & $12.2 \%$ & $\begin{array}{c}63.6 \% \\
(40.8-82.0)\end{array}$ & $\begin{array}{c}76.9 \% \\
(72.6-80.7)\end{array}$ & $\begin{array}{c}12.2 \% \\
(7.4-19.4)\end{array}$ & $\begin{array}{c}97.7 \% \\
(95.5-98.8)\end{array}$ & 2.8 & $\begin{array}{c}5.82 \\
(2.38-14.27)\end{array}$ & \\
\hline \multirow[t]{2}{*}{$\begin{array}{l}\text { ESC } 2019 \\
\text { intermediate-high-risk" }\end{array}$} & $\mathrm{hs} \operatorname{TnT} \geqslant 14 \mathrm{pg} \cdot \mathrm{mL}^{-1}$ & $32.2 \%$ & $8.8 \%$ & $\begin{array}{c}59.1 \% \\
(36.7-78.5)\end{array}$ & $\begin{array}{c}69.1 \% \\
(64.5-73.4)\end{array}$ & $\begin{array}{c}8.8 \% \\
(5.2-14.4)\end{array}$ & $\begin{array}{c}97.1 \% \\
(94.6-98.5\end{array}$ & 1.9 & $\begin{array}{c}3.23 \\
(1.35-7.74)\end{array}$ & $\begin{array}{c}0.03 \text { (SE } \\
0.07) \quad p=0.68\end{array}$ \\
\hline & $\mathrm{hs} T \mathrm{nl}>16 \mathrm{pg} \cdot \mathrm{mL}^{-1}$ & $29.6 \%$ & $9.6 \%$ & $\begin{array}{c}59.1 \% \\
(36.7-78.5)\end{array}$ & $\begin{array}{c}71.9 \% \\
(67.3-76.0)\end{array}$ & $\begin{array}{c}9.6 \% \\
(5.7-15.7)\end{array}$ & $\begin{array}{c}97.2 \% \\
(94.8-98.5)\end{array}$ & 2.1 & $\begin{array}{c}3.69 \\
(1.54-8.85)\end{array}$ & \\
\hline
\end{tabular}

HsTnl: high-sensitivity troponin I; hsTnT: high-sensitivity troponin $\mathrm{T} ; \mathrm{Cl}$ : confidence interval; PPV: positive predictive value; NPV: negative predictive value; LR+: positive likelihood ratio;

OR: odds ratio; NRI: net reclassification improvement; SE: standard error; ESC: European Society of Cardiology. \#: Compared to ESC 2019 low/intermediate-low risk. 


\section{Discussion}

In the present study we evaluated the prognostic value of hsTnI in normotensive PE patients and established a disease-specific hsTnI cut-off value for risk stratification. Our findings obtained in 459 patients can be summarised as follows: 1) hsTnI concentrations $>16 \mathrm{pg} \cdot \mathrm{mL}^{-1}$ were predictive of both, an in-hospital adverse outcome and all-cause mortality; 2) sex-specific cut-off values do not seem necessary for risk stratification of acute PE; and 3) if hsTnI and hsTnT were used to risk-stratify patients according to the modified FAST score and ESC 2019 algorithm, no difference in the prognostic performance was observed.

High-sensitivity troponin assays are recommended for the diagnosis of myocardial infarction in current European and American guidelines and have seen high adoption rates in clinical practice [10, 24]. Although these contemporary assays improve the diagnostic accuracy at low analyte concentrations, their increase in sensitivity comes at the disadvantage of reduced specificity [25]. This emphasises the importance of cut-off values tailored to specific disease states and applications. In patients with suspected acute coronary syndrome (ACS), troponin concentrations below the 99th percentile upper reference limit (URL) are used to rule out non-ST-elevation ACS [10, 24]. In contrast, troponin is not measured for diagnostic purposes in patients with acute PE but contributes to risk stratification after the diagnosis has been established. Hence, the diagnostic troponin threshold for myocardial infarction might not be identical to the optimal cut-off value for risk stratification in acute PE.

Data on disease-specific troponin I cut-off values for risk stratification of acute PE are limited, as most previous reports relied on the 99th percentile URL $[7,26]$. In addition, the majority of investigations relied on conventional troponin I assays rather than highly sensitive ones $[7,26]$. Until now, the only evidence supporting the use of hsTnI for risk stratification of acute PE comes from a single study including 65 patients only [12]. Importantly, this study investigated the cut-off value suggested for evaluation of ACS at the time of publication $\left(100 \mathrm{pg} \cdot \mathrm{mL}^{-1}\right)$ [12], that is much higher than contemporary thresholds [20].

Our study adds substantially to these previous investigations. Based on our results, a hsTnI cut-off value of $16 \mathrm{pg} \cdot \mathrm{mL}^{-1}$ provides high sensitivity $(86.4 \%)$ and a high negative predictive value (98.7\%) for the prediction of adverse outcomes and thus offers better prognostic performance than cut-off values corresponding to the 99th percentile URL [19], the sex-specific 99th percentile URL [20] and the cut-off value suggested by WALTER et al. [12]. Of note, the identified optimal hsTnI cut-off value is lower than the 99th percentile URL reported by the manufacturer [19] but identical to the 99th percentile URL reported for females and higher than cut-off values recommended to rule out non-ST-elevation ACS [10] and for risk stratification of asymptomatic patients with cardiovascular disease [19]. Thus, potentially more male patients with PE will be classified as intermediate-high risk, requiring hospital admission and monitoring, which might increase safety and is very unlikely to cause harm. However, as a lower cut-off value may lead to more false positive results (and hence potentially overtreatment), the costs and effectiveness associated with a higher number of PE patients classified as intermediate-high risk require additional investigation in further studies.

Interestingly, although sex-specific differences of hsTnI values have been described for patients with myocardial infarction [20], we did not observe such differences in our cohort of PE patients. Optimal cut-off values for female and male patients were similar $\left(17 \mathrm{pg} \cdot \mathrm{mL}^{-1}\right.$ and $19 \mathrm{pg} \cdot \mathrm{mL}^{-1}$, respectively) and hence, do not indicate the use of sex-specific cut-off values for risk stratification of acute PE.

The recently published ESC 2019 guidelines emphasise the central role of troponin elevation for risk stratification in acute PE and consider the troponin subunits I and T as equally suitable for this purpose [3]. This is based on the results of two meta-analyses concluding that the prognostic value of increased troponin concentrations in patients with acute PE is independent regardless of whether troponin $\mathrm{T}$ or I subunits are measured [7, 26]. The findings of our study are consistent with these previous reports and add to the evidence that hsTnI and hsTnT have comparable prognostic performance. Hence, clinicians are free to choose either assay and troponin subunit for risk stratification of normotensive PE in clinical practice.

\section{Limitations}

The present study is a post hoc analysis of a prospective single-centre cohort, thus the generalisability of the study findings may be limited. In particular, the high prevalence of comorbidities such as cancer (24.0\%) and renal insufficiency (29.2\%) should be taken into account when interpreting the present study findings. A considerable number of patients were excluded from the study due to missing hsTnI measurements at presentation. However, the comparison of patients with and without hsTnI measurements provided in in the supplementary table S1 did not reveal relevant differences between the two groups. Finally, due to the small number of events, the ability to perform robust multivariable analyses was limited. 


\section{Conclusion}

Our findings confirm the prognostic value of hsTnI for risk stratification of patients with normotensive PE. Elevation of hsTnI concentrations exceeding a cut-off value of $16 \mathrm{pg} \cdot \mathrm{mL}^{-1}$ predict in-hospital adverse outcome and all-cause mortality in both male and female patients. Furthermore, our results suggest that hsTnI and hsTnT have similar prognostic value and can be used interchangeably for risk stratification.

Acknowledgements: The authors thank Christian Thode and Daniela Brehm (amedes MVZ wagnerstibbe, Göttingen, Germany) for performing the biomarker measurements. This research was supported by the UMG Biobank as a core facility of the University Medical Center Göttingen, Germany, by provision of quality assured biomaterial. This publication is part of the medical doctoral thesis of Niklas Guddat.

Conflict of interest: M. Ebner has nothing to disclose. N. Guddat has nothing to disclose. K. Keller has nothing to disclose. M.C. Merten has nothing to disclose. M.H. Lerchbaumer reports personal fees from Siemens Healthineers outside the submitted work. G. Hasenfuß reports personal fees from AstraZeneca, Berlin Chemie, Corvia, Impulse Dynamics, Novartis, Servier, Vifor Pharma and Springer International Publishing AG outside the submitted work. S.V. Konstantinides reports a grant from the German Federal Ministry of Education and Research (BMBF 01EO1503) during the conduct of the study; grants and personal fees from Bayer, Boehringer Ingelheim and Daiichi-Sankyo, personal fees from MSD, grants and personal fees from Pfizer-Bristol-Myers Squibb, and grants from Actelion, outside the submitted work. M. Lankeit reports grants from the German Federal Ministry of Education and Research (BMBF 01EO1503) and grants from BRAHMS GmbH, part of Thermo Fisher Scientific, during the conduct of the study; and personal fees from Actelion, Bayer, Daiichi-Sankyo, MSD, Pfizer-Bristol-Myers Squibb and BRAHMS GmbH outside the submitted work.

Support statement: The work of Karsten Keller, Stavros V. Konstantinides and Mareike Lankeit was supported by the German Federal Ministry of Education and Research (BMBF 01EO1503). The authors are responsible for the contents of this publication. BRAHMS GmbH, part of Thermo Fisher Scientific, Hennigsdorf/Berlin, Germany, provided financial support for biomarker measurements. The sponsor was not involved in biomarker measurements, statistical analyses or writing of the manuscript, nor had any influence on the scientific contents of this publication. Funding information for this article has been deposited with the Crossref Funder Registry.

\section{References}

1 Wendelboe AM, Raskob GE. Global burden of thrombosis: epidemiologic aspects. Circ Res 2016; 118: 1340-1347.

2 Keller K, Hobohm L, Ebner M, et al. Trends in thrombolytic treatment and outcomes of acute pulmonary embolism in Germany. Eur Heart J 2019; 41: 522-529.

3 Konstantinides SV, Meyer G, Becattini C, et al. 2019 ESC Guidelines for the diagnosis and management of acute pulmonary embolism developed in collaboration with the European Respiratory Society (ERS): The Task Force for the diagnosis and management of acute pulmonary embolism of the European Society of Cardiology (ESC). Eur Respir J 2019; 54: 1901647.

4 Ribeiro A, Lindmarker P, Juhlin-Dannfelt A, et al. Echocardiography Doppler in pulmonary embolism: right ventricular dysfunction as a predictor of mortality rate. Am Heart J 1997; 134: 479-487.

5 Vonk Noordegraaf A, Westerhof BE, Westerhof N. The relationship between the right ventricle and its load in pulmonary hypertension. J Am Coll Cardiol 2017; 69: 236-243.

6 Harjola VP, Mebazaa A, Celutkiene J, et al. Contemporary management of acute right ventricular failure: a statement from the Heart Failure Association and the Working Group on Pulmonary Circulation and Right Ventricular Function of the European Society of Cardiology. Eur J Heart Fail 2016; 18: 226-241.

7 Becattini C, Vedovati MC, Agnelli G. Prognostic value of troponins in acute pulmonary embolism: a meta-analysis. Circulation 2007; 116: 427-433.

8 Kaeberich A, Seeber V, Jimenez D, et al. Age-adjusted high-sensitivity troponin $\mathrm{T}$ cut-off value for risk stratification of pulmonary embolism. Eur Respir J 2015; 45: 1323-1331.

9 Hobohm L, Hellenkamp K, Hasenfuss G, et al. Comparison of risk assessment strategies for not-high-risk pulmonary embolism. Eur Respir J 2016; 47: 1170-1178.

10 Roffi M, Patrono C, Collet JP, et al. 2015 ESC Guidelines for the management of acute coronary syndromes in patients presenting without persistent ST-segment elevation: Task Force for the Management of Acute Coronary Syndromes in Patients Presenting without Persistent ST-Segment Elevation of the European Society of Cardiology (ESC). Eur Heart J 2016; 37: 267-315.

11 Lankeit M, Friesen D, Aschoff J, et al. Highly sensitive troponin T assay in normotensive patients with acute pulmonary embolism. Eur Heart J 2010; 31: 1836-1844.

12 Walter T, Apfaltrer P, Weilbacher F, et al. Predictive value of high-sensitivity troponin I and D-dimer assays for adverse outcome in patients with acute pulmonary embolism. Exp Ther Med 2013; 5: 586-590.

13 Ebner M, Kresoja KP, Keller K, et al. Temporal trends in management and outcome of pulmonary embolism: a single-centre experience. Clin Res Cardiol 2020; 109: 67-77.

14 Lankeit M, Friesen D, Schafer K, et al. A simple score for rapid risk assessment of non-high-risk pulmonary embolism. Clin Res Cardiol 2013; 102: 73-80.

15 Torbicki A, Perrier A, Konstantinides S, et al. Guidelines on the diagnosis and management of acute pulmonary embolism: the Task Force for the Diagnosis and Management of Acute Pulmonary Embolism of the European Society of Cardiology (ESC). Eur Heart J 2008; 29: 2276-2315.

16 Konstantinides SV, Torbicki A, Agnelli G, et al. 2014 ESC guidelines on the diagnosis and management of acute pulmonary embolism. Eur Heart J 2014; 35: 3033-3069.

17 Raskob GE, van Es N, Segers A, et al. Edoxaban for venous thromboembolism in patients with cancer: results from a non-inferiority subgroup analysis of the Hokusai-VTE randomised, double-blind, double-dummy trial Lancet Haematol 2016; 3: e379-e387.

18 Lankeit M, Jimenez $\mathrm{D}$, Kostrubiec $\mathrm{M}$, et al. Predictive value of the high-sensitivity troponin $\mathrm{T}$ assay and the simplified Pulmonary Embolism Severity Index in hemodynamically stable patients with acute pulmonary embolism: a prospective validation study. Circulation 2011; 124: 2716-2724. 
19 Beshiri A, Ravalico T. A New Paradigm for Acute Coronary Syndrome: High Sensitive Troponin. www. corelaboratory.abbott/sal/whitePaper/CR_ADD-00001076_ARCHITECT_hsTnI_WhitePaper_072613.pdf Date last accessed: Nov 14, 2019. Date last updated: 2013.

20 Shah ASV, Anand A, Strachan FE, et al. High-sensitivity troponin in the evaluation of patients with suspected acute coronary syndrome: a stepped-wedge, cluster-randomised controlled trial. Lancet 2018; 392: 919-928.

21 Lankeit M, Jimenez D, Kostrubiec M, et al. Validation of N-terminal pro-brain natriuretic peptide cut-off values for risk stratification of pulmonary embolism. Eur Respir J 2014; 43: 1669-1677.

22 Pencina MJ, D’Agostino RB, Sr., D'Agostino RB, Jr., et al. Evaluating the added predictive ability of a new marker: from area under the ROC curve to reclassification and beyond. Stat Med 2008; 27: 157-172.

23 Meyer G, Vicaut E, Danays T, et al. Fibrinolysis for patients with intermediate-risk pulmonary embolism. $N$ Engl J Med 2014; 370: 1402-1411.

24 Amsterdam EA, Wenger NK, Brindis RG, et al. 2014 AHA/ACC Guideline for the Management of Patients with Non-ST-Elevation Acute Coronary Syndromes: a report of the American College of Cardiology/American Heart Association Task Force on Practice Guidelines. J Am Coll Cardiol 2014; 64: e139-e228.

25 Januzzi JL, Jr. What to expect when measuring high-sensitivity troponin: practical advice for clinicians. J Am Coll Cardiol 2015; 65: 1665-1667.

26 Bajaj A, Saleeb M, Rathor P, et al. Prognostic value of troponins in acute nonmassive pulmonary embolism: a meta-analysis. Heart Lung 2015; 44: 327-334. 